\title{
Lessons Learned from a DoD and Virginia Data Sharing Pilot using NSSP ESSENCE
}

\author{
Erin E. Austin ${ }^{1}$, Paul E. Lewis ${ }^{2}$, Arden Norfleet ${ }^{1}$, Jamaal Russell ${ }^{2}$ \\ ${ }^{1}$ Virginia Department of Health, Richmond, Virginia, United States, ${ }^{2}$ Armed Forces Health Surveillance Branch, Silver Spring, Maryland, United States \\ Objective
}

This panel will focus on the experiences from the Department of Defense (DoD) and Virginia Department of Health (VDH) data sharing project using the National Syndromic Surveillance Program (NSSP) ESSENCE and will discuss lessons learned, challenges, and recommendations within the following areas: 1) data sharing authority, 2) coordination and implementation of data sharing with a focus on personnel, training, and managing access and 3) communication between local, state, and federal agencies.

\section{Introduction}

The DoD and VDH both maintain local ESSENCE installations to monitor the health status of their military and civilian populations, respectively, and submit syndromic surveillance data to the NSSP ESSENCE to foster data sharing and collaborative initiatives among public health entities. Military Treatment Facilities (MTFs), housed on DoD installations, provide healthcare to all service members and their beneficiaries stationed in the area. Service members and their beneficiaries represent a substantial portion of the local community and interact with the civilian population throughout daily activities. Sharing syndromic surveillance data between DoD and public health jurisdictions can provide public health situational awareness among both civilian and military populations to support disease surveillance. DoD and VDH engaged in a pilot project to develop processes and procedures for data sharing, data access, and communication with the aim they can serve as best practices for other jurisdictions seeking to share syndromic surveillance data with DoD.

\section{Description}

The pilot project began in June 2018 with the Centers for Disease Control and Prevention (CDC) NSSP team providing technical support. NSSP ESSENCE users from the VDH state and local health departments across nine Virginia city/counties participated in the project. VDH shared syndromic surveillance data from 34 healthcare facilities (17 urgent cares, 3 emergency care centers, and 14 hospitals) with DoD, which shared syndromic surveillance data from 18 MTFs (16 clinics and 2 hospitals) in Virginia. To standardize the analysis of syndromic surveillance data and use of NSSP ESSENCE across project participants, myESSENCE tabs were created and shared by between VDH and DoD. The goal was to facilitate and enhance communication between local public health departments and their DoD counterparts through the sharing of syndromic surveillance data.

\section{Acknowledgement}

Thank you to Mike Coletta and the NSSP Team at CDC for their support. 